\title{
EXPECTATIVAS DOS KEY-USERS QUANTO À ADOÇÃO DE SISTEMA INTEGRADO DE CONTROLE FINANCEIRO
}

\author{
KEY-USERS' EXPECTATIONS REGARDING THE ADOPTION OF AN \\ INTEGRATED FINANCIAL CONTROL SYSTEM
}

\begin{abstract}
Aline Vieira Malanovicz
Resumo: Entre os fatores que podem motivar o sucesso dos projetos de desenvolvimento e aplicação de tecnologia estão as expectativas e a aceitação da implantação de novos sistemas de informação. Nessas condições, o objetivo desta pesquisa é identificar, no contexto de uma empresa pública do setor financeiro, quais são as expectativas dos usuárioschaves em relação à implantação de um novo sistema de informação, analisando-as segundo o modelo da Teoria Unificada de Aceitação e Uso da Tecnologia (UTAUT). A coleta de dados foi realizada através de entrevistas johpor telefone e de questionário enviado por e-mail em março de 2019 para os sujeitos selecionados - usuários-chaves dos processos que devem ser suportados pelo novo sistema. Como resultado, foram identificadas, na pesquisa, as variáveis do modelo UTAUT para intenção de uso, quais sejam: expectativa de desempenho (confiabilidade das informações, utilidade do sistema, agilidade na realização das tarefas, melhoria geral no trabalho), expectativa de esforço (interação com o novo sistema, agilidade no uso do sistema, facilidade de uso do sistema, dificuldade em aprender) e influência social (opinião das pessoas influentes, prestígio das pessoas que utilizam o sistema, auxílio do gestor, apoio geral da empresa). Também foram identificadas variáveis moderadoras (sendo elas: gênero, idade, experiência, voluntariedade) e expectativas positivas quanto às condições facilitadoras (suporte para a escolha do sistema, suporte técnico e operacional na implementação, suporte técnico no uso). Conclui-se que os resultados qualitativos parecem se adequar ao modelo. $\mathrm{O}$ estudo dos fatores referentes ao efetivo comportamento de uso é proposto como interesse para pesquisa futura.
\end{abstract}

Palavras-chave: Gestão de T.I. Expectativas dos usuários-chaves. UTAUT.

\begin{abstract}
Factors that can motivate the success of the projects of development and application of technology include expectations and acceptance of the implantation of new information systems. The objective of this research is to identify, in the context of a public company in the financial sector, what are the expectations of key-users in relation to the implementation of a new information system, analyzing them according to the model of the Unified Theory of Acceptance and Use of Technology (UTAUT). Data collection was carried out through telephone interviews and a questionnaire sent by email in March 2019 to the selected subjects - key-users of the processes that must be supported by the new system. As a result, the variables identified in the research were the UTAUT model for Intention to Use, namely: Performance Expectancy (Reliability of information, System utility, Agility in performing tasks, General improvement in work), Expectation of Effort (Interaction with the new system, Agility in the use of the system, Ease of use of the system, Difficulty in learning), Social Influence (Opinion of influential people, Prestige of the people who use the system, Manager assistance, General company support). Moderating Variables (namely: Gender, Age, Experience, Voluntariness) and positive expectations regarding the Facilitating Conditions (Support for choosing the system, Technical and operational support in implementation, Technical support in use) were also identified. It is concluded that the qualitative results seem to fit the model. The study of the factors referring to effective Use Behavior is proposed as an interest for future research.
\end{abstract}

Keywords: Information technology management. Key-user expectations. UTAUT.

'Doutora em Administração, Universidade Federal do Rio Grande do Sul - UFRGS. Porto Alegre, RS - Brasil. malanovicz@gmail.com

\section{Cite como}

American Psychological Association (APA)

Malanovicz, A. V. (2020, jul./dez.). Expectativas dos key-users quanto à adoção de sistema integrado de controle financeiro. Revista Inovação, Projetos e Tecnologias, São Paulo, 8(2), 139-157.

https://doi.org/10.5585/iptec.v8i2.16735. 


\section{Introdução}

Entre os temas mais pesquisados na área de inovação estão o desenvolvimento e a aplicação de tecnologias (Vilela, Alves, Macedo, Werneck, \& Ferreira, 2018). A efetiva implantação de sistemas e de tecnologias nas organizações apresenta desafios para os projetos e para uma gestão inovadora (Bozzo, Freitas, \& Martens, 2019; Ren, 2019; Webster, \& Gardner, 2019; Hoppen, Klein, \& Rigoni, 2017). Por exemplo, a falta de análise das variáveis envolvidas na adoção de um novo sistema pode expor qualquer empresa ao risco de desperdiçar parte ou todo o investimento realizado na implantação de novas tecnologias (Marques, Behr, \& Malanovicz, 2018). Em empresas pequenas e médias, os principais motivos que as têm levado a adotar a tecnologia de informação estão relacionados a pressões externas que elas enfrentam e à existência de um ambiente organizacional favorável (Lunardi, Dolci, \& Dolci, 2017; Nguyen, Newby, \& Macaulay, 2015).

Por um lado, uma das causas do fracasso da implantação de novos sistemas é a sua não aceitação, apontada como um dos fatores de resistência envolvidos no processo de mudança (Santos, \& Jesus, 2005; Angonese, \& Lavarda, 2014; Hoppen et al., 2017). A resistência à tecnologia e os fatores que enfraquecem a cooperação para seu uso representam desafios insuperáveis para a adoção de um sistema (Hoppen et al., 2017). Para a adesão a sistemas financeiros e contábeis, o processo de mudança enfrenta resistências internas por insegurança, falta de confiança, de conhecimento e aceitação de rotinas (Angonese, \& Lavarda, 2014).

Por outro lado, entre os fatores que podem motivar o sucesso dos projetos estão as expectativas e a aceitação da implantação de novos sistemas de informação (Machado \& Martens, 2015). Para sistemas financeiros, especificamente, o design e a segurança são relevantes para o desempenho das suas funcionalidades, bem como a facilidade de uso e a utilidade percebidas o são para a intenção de uso do sistema (Lai, 2017). Por exemplo, a correta entrada de dados no sistema tem uma importância fundamental para a sobrevivência de uma tecnologia adotada numa organização (Markard, Wirth, \& Truffer, 2016; Ren, 2019).

Para um sistema ser efetivo para uma empresa, é preciso que ele seja aceito e utilizado pelos usuários (Venkatesh, Morris, Davis, \& Davis, 2003). A legitimação intraorganizacional da adoção do sistema é atualmente uma perspectiva explorada em pesquisas sobre quais fatores determinam o sucesso ou o fracasso da adesão a tecnologias (Ren, 2019). Em empresas do setor financeiro, por exemplo, o sistema de controle financeiro representa seu core business e, por isso, o projeto de implantação de um sistema como esse é delicado, pois demanda investimentos altos e sua não aceitação pode comprometer tudo isso. 
O sucesso de qualquer projeto depende do eficiente monitoramento e do constante gerenciamento das expectativas dos key-users (usuários-chaves) e demais stakeholders (partes interessadas) (Project Management Institute, 2017). A realização de pesquisas de opinião com tais pessoas é a técnica que permite entender, desde o início do projeto, quais são os interesses e as expectativas delas para, ao longo de sua execução, melhor direcionar sua condução, ampliando e reforçando a intenção de uso do sistema, bem como a satisfação e o engajamento (Project Management Institute, 2017). Quanto antes se difundirem expectativas favoráveis (mas realistas) sobre o sistema, maior é a chance de ser positiva a atitude das pessoas em relação à sua adoção (Souza, \& Saccol, 2011).

Entretanto, embora seja uma prática recomendada na literatura de projetos (Project Management Institute, 2017), nem todas as empresas que iniciaram a implantação de sistemas novos realizaram essas avaliações. Alguns casos de fracasso pesquisados (Santos, \& Jesus, 2005; Hoppen et al., 2017; Malanovicz, 2017; Bozzo et al., 2019), especialmente de sistemas financeiros e contábeis (Angonese, \& Lavarda, 2014; Lai, 2017), relatam a resistência à mudança (baixa intenção de uso do sistema), consequência da falta de gestão das expectativas dos stakeholders, como um dos fatores que contribuíram para o insucesso do projeto.

Portanto, para alcançar o sucesso na implantação de sistemas, é relevante conhecer as expectativas e a aceitação dos seus futuros usuários. Entende-se, então, que um relato dessa intervenção pode contribuir para que outros gestores de outras empresas possam replicá-la. Um modelo teórico já validado para a avaliação da aceitação dos sistemas é a Teoria Unificada de Aceitação e Uso da Tecnologia (Unified Theory of Acceptance and Use of Technology UTAUT) (Venkatesh et al., 2003). A adoção desse modelo pode contribuir para estimular o uso do sistema e para evitar problemas que comprometam sua implantação (Marques et al., 2018).

Finalmente, o objetivo deste estudo é identificar, no contexto de uma empresa pública do setor financeiro, quais são as expectativas dos usuários-chaves em relação à implantação de um novo sistema de informação, analisando-as segundo o modelo UTAUT. Para tanto, este artigo apresenta, além desta introdução, uma revisão teórica que comenta fatores de insucesso em projetos de implantação de sistemas e explica o modelo UTAUT, seguida pela seção que expõe o percurso metodológico da pesquisa realizada através de entrevistas por telefone e de questionário enviado via e-mail, pelos resultados e pela conclusão. 


\section{Revisão teórica}

Entre os principais fatores que afetam a adesão de uma tecnologia por uma organização existem os chamados fatores técnicos, organizacionais e técnico-organizacionais (Ren, 2019). As dificuldades para a adoção de tecnologias inovadoras podem ter relação com o conceito de prontidão para a inovação, tanto da tecnologia quanto da própria organização (Webster, \& Gardner, 2019). Para sistemas financeiros, como foi citado, são essenciais para a intenção de uso a segurança e o design, além da facilidade de uso e da utilidade percebida (Lai, 2017). Para sistemas integrados, especificamente, fatores críticos de sucesso incluem o envolvimento do usuário, o apoio da direção, a definição clara de necessidades, o planejamento adequado e, destacadamente, as expectativas realistas:

Os sistemas integrados geralmente são considerados como solução para o atendimento de todos os requisitos impostos pelo ambiente da organização e pelas necessidades e expectativas dos usuários, porém, é pouco provável que tais requisitos combinem perfeitamente com o sistema. Criam-se então as diferenças ou discrepâncias entre a funcionalidade do sistema e os requisitos da empresa para o sistema. Tais discrepâncias são resolvidas basicamente de quatro maneiras: ou adapta-se o pacote, ou adaptam-se os processos da organização, ou adaptam-se tanto o pacote como os processos, ou não se adapta nem o pacote nem o processo, optando-se pela convivência com a discrepância. (Souza, \& Saccol, 2011, p. 71).

Pesquisas sobre casos de insucesso na implantação de sistemas existem na literatura de inovação, projetos e tecnologias. Há também exemplos de fracasso na introdução de tecnologias entre produtores de café (Santos, \& Jesus, 2005), adoção e difusão de plataforma para comunidades virtuais de negócios (Hoppen et al., 2017), além de muitas dificuldades iniciais em casos de implantação de startups de Internet of Things (Bozzo et al., 2019).

Entre os principais fatores de insucesso de projetos de implantação de sistemas estão: a resistência à mudança por parte dos funcionários, a dificuldade para mudança cultural na empresa, a desmotivação/desinteresse das pessoas com o projeto, a dificuldade de aprendizagem da ferramenta, os treinamentos deficientes, a falta de suporte técnico do sistema implantado e a frustração das expectativas (por vezes não realistas) quanto ao novo sistema (Malanovicz, 2017). Para sistemas integrados, várias manifestações de relutância podem ocorrer, sejam por recusa, confusão, medo, ansiedade, falta de confiança, inércia (Souza, \& Saccol, 2011). Para sistemas financeiros e contábeis, pode haver objeção por fatores como falta de confiança, falta de conhecimento, aceitação de rotinas e insegurança (Angonese, \& Lavarda, 2014). 
Em relação às expectativas dos usuários-chaves, em especial a sua intenção de uso do sistema, sua avaliação é necessária para embasar decisões relativas, por exemplo, ao conteúdo e ao direcionamento do processo de comunicação do projeto, às previsões de treinamentos, à cobertura dos testes e ao sequenciamento das fases de implantação dos módulos do sistema. Essa apreciação deve mostrar aos gestores quais aspectos do novo sistema precisam ser demonstrados com maior detalhamento (a exemplo de usabilidade, integridade, validações) e quais funcionalidades as pessoas esperam poder utilizar primeiro (a exemplo de simulações de cálculos de parcelas de financiamento). Esse conhecimento deve influenciar a implantação no sentido de adequar, dentro das possibilidades do sistema, as preferências dos usuários-chaves na lógica de estabelecimento dos módulos da solução contratada e definir quais aspectos receberão maior destaque no material informativo para comunicação interna.

O tratamento pró-ativo das expectativas dos usuários-chaves permite que elas sejam conhecidas, alinhadas, ajustadas, direcionadas pela equipe do projeto, especialmente no processo de comunicação e de treinamentos, evitando o desperdício de recursos (financeiros, de tempo, de trabalho de pessoas, de subutilização de funções do sistema) por mal-entendidos sobre o objetivo do projeto ou das etapas da implantação; e amplia a intenção de uso do sistema. Como essas expectativas podem se modificar no decorrer do projeto, seu monitoramento deve ser realizado por meio da repetição da pesquisa em outros momentos, por exemplo, após alguns meses de implantação da primeira etapa, às vésperas da segunda fase e ao final para que seu atendimento seja verificado ou não.

Considerando situações assim, pesquisadores tentam explicar fatores que influenciam o comportamento das pessoas e a sua aceitação dos sistemas. O modelo da Teoria Unificada de Aceitação e Uso da Tecnologia (Unified Theory of Acceptance and Use of Technology UTAUT) reuniu aspectos comuns a oito teorias (Theory of Reasoned Action (TRA), Theory of Planned Behavior (TPB), Technology Acceptance Model (TAM), Motivational Model (MM), Combined TAM and TPB (C-TAM-TPB), Model of PC Utilization (MPCU), Innovation Diffusion Theory (IDT) e Social Cognitive Theory (SCT)) (Venkatesh et al., 2003).

O modelo de pesquisa da UTAUT (Figura 1) apresenta quatro variáveis independentes (expectativa de desempenho, expectativa de esforço, influência social e condições facilitadoras), uma mediadora (intenção de uso), uma dependente (comportamento de uso) e quatro moderadoras (gênero, idade, experiência e voluntariedade) (Venkatesh et al., 2003): 
Figura 1 - Modelo de pesquisa da teoria unificada de aceitação e uso da tecnologia

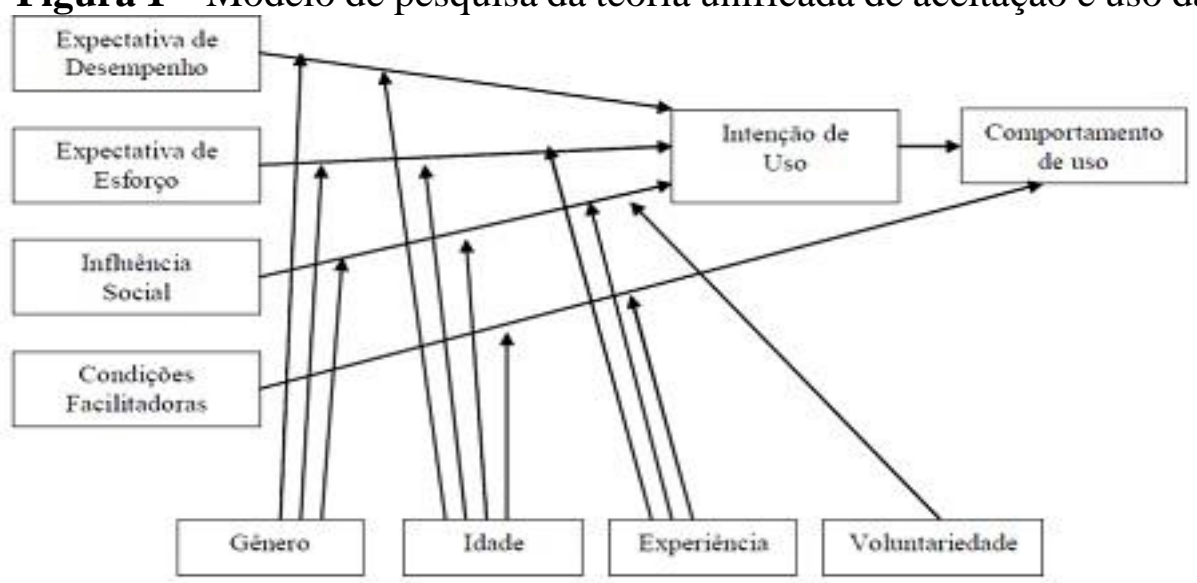

Fonte: Venkatesh et al. (2003).

- Expectativa de desempenho: grau em que uma pessoa crê que a utilização do sistema irá ajudá-la a ganhar desempenho no trabalho. Moderado por gênero e idade, é o mais forte preditor da intenção de uso, especialmente em homens mais jovens;

- Expectativa de esforço: grau de facilidade associado ao uso do sistema. Moderado por gênero, idade e experiência, é maior em mulheres mais jovens e menos experientes;

- Influência social: grau em que uma pessoa percebe que outras pessoas importantes para ela creem que o novo sistema deva ser usado. Tal aspecto é moderado pelas quatro condições, é mais forte em mulheres mais velhas e menos experientes e só é relevante onde o uso do sistema é obrigatório;

- Condições facilitadoras: grau em que uma pessoa crê que uma infraestrutura técnicoorganizacional existe para apoiar a utilização do sistema. É moderado por idade e experiência, sendo mais forte em pessoas mais velhas e mais experientes (influencia no comportamento de uso, não na intenção de uso).

A variável de gênero modera a expectativa de desempenho, a expectativa de esforço e a influência social. A idade modera todas as quatro variáveis independentes. A experiência modera a expectativa de esforço, a influência social e as condições facilitadoras e, por fim, a voluntariedade modera a influência social. A variável mediadora (intenção de uso) é o que se pretende avaliar em uma pesquisa de início de projeto; as condições facilitadoras não influenciam a intenção de uso, mas podem ser investigadas desde já para a avaliação do comportamento de uso, a ser realizada em futuramente. 


\section{Percurso metodológico}

Esta pesquisa tem natureza qualitativa e caráter exploratório (Gil, 2008). O cenário de estudo é uma empresa pública de grande porte (500 funcionários) do setor financeiro, cuja atuação se dá no Sul do Brasil, no segmento de financiamentos de médio e longo prazo. Atualmente, inicia-se, nela, um projeto de implantação de um sistema de informação. Este trabalho compõe uma fase inicial de uma série de pesquisas a serem realizadas durante a iniciativa e procura identificar as expectativas dos usuários-chaves em relação à adoção desse novo sistema, analisando-as segundo o modelo UTAUT (Venkatesh et al., 2003).

Os processos do núcleo do negócio da empresa (core business), no encarteiramento e controle financeiro (back office), são ainda atendidos por um sistema legado dos anos 1970 que roda em mainframe e apresenta sérias dificuldades de manutenção. Por razões técnicas e negociais, a organização precisa atualizar a tecnologia dos seus processos de controle financeiro. Por essa razão, optou pela aquisição, em processo licitatório, de um novo sistema, o qual contempla processos de controle financeiro de financiamentos, cobrança, tesouraria, contabilização, auditoria e compliance, recuperação de crédito e demais fluxos de trabalho e tecnologia de informação da área financeira.

Os usuários-chaves (key-users), ou seja, as pessoas que detêm o conhecimento técnico e o poder de decisão sobre o modo de execução dos citados processos, somam 20 pessoas na empresa, incluindo analistas técnicos seniores e chefias de setores e departamentos. Um universo maior, de cerca de 60 pessoas, abrangeria todos os futuros usuários do novo sistema, incluindo analistas juniores e assistentes administrativos.

A coleta de dados foi realizada por meio de questionário enviado por e-mail em março de 2019 - para todos os 60 indivíduos mencionados, inicialmente. Do total de respondentes (18), identificou-se que eram todos usuários-chaves, não havendo respostas de assistentes nem de juniores. Considerou-se, então, o recorte de público da pesquisa como sendo somente os keyusers, dos quais se obteve uma taxa de resposta de $90 \%$ (18/20), o que se mostra representativo desse público e, assim, contribui para a validade dos resultados encontrados.

O questionário elaborado (Figura 2) resultou da adaptação do instrumento já adotado como roteiro de entrevistas em outro trabalho (Marques et al., 2018), mas, aqui, focado no sistema de controle financeiro (que guarda características de porte, integração, modularidade e workflow semelhantes às dos sistemas ERP pesquisados no estudo citado) e nas expectativas e na intenção de uso (pré-implantação) dos key-users - não em suas percepções (pósimplantação) devido ao momento atual do projeto. Destaca-se a importância da coleta das 
impressões sobre expectativas para intenção de uso no início para que não haja confusão entre memórias antigas e novas ou entre expectativas e percepções (Marques et al., 2018).

Figura 2 - Instrumento de coleta de dados via questionário por e-mail

Prezados, peço a sua colaboração nesta pesquisa sobre Adoção de Tecnologia.

É só responder umas perguntinhas sobre as suas expectativas em relação ao NovoFN.

Não tem certo nem errado: a sua opinião é o que vale. :)

Basta uma linha ou duas de texto para cada resposta. ;-)

Pode ser alguma comparação com a situação atual, ou outra forma.

Os resultados serão muito úteis para o projeto do NovoFN.

E também para algum eventual futuro artigo de pesquisa. ;-)

A base teórica do tema (modelo UTAUT) está resumida no anexo.

Poderia ser para esta sexta (29.03.2019)?

Muito muito grata!!!

1. Quais são as suas expectativas a respeito da confiabilidade das informações no sistema?

2. Quais são as suas expectativas em relação à utilidade do sistema no seu trabalho?

3. Quais são as suas expectativas quanto à agilidade na realização das tarefas com o uso do sistema?

4. Quais são as suas expectativas sobre a melhoria geral que o sistema agregará ao trabalho?

5. Quais são as suas expectativas referentes ao esforço necessário para interagir com o sistema?

6. Quais são as suas expectativas com relação ao esforço necessário para ter agilidade no uso do sistema?

7. Quais são as suas expectativas quanto à facilidade de uso do sistema?

8. Quais são as suas expectativas sobre o grau de dificuldade em aprender a trabalhar com o sistema?

9. Quais são as suas expectativas referentes à opinião das pessoas, que influenciam seu comportamento, quanto a utilizar ou não o sistema?

10. Quais são as suas expectativas referentes ao prestígio das pessoas que devem utilizar o sistema?

11. Quais são as suas expectativas quanto ao auxílio do gestor da sua área na utilização no sistema?

12. Quais são as suas expectativas em relação ao apoio geral da empresa na utilização do sistema?

13. Quais são as suas expectativas quanto ao suporte necessário para a escolha do sistema?

14. Quais são as suas expectativas referentes ao suporte técnico e operacional especializado necessário na implantação do sistema?

15. Quais são as suas expectativas a respeito do suporte técnico necessário para resolver problemas no uso do sistema?

\begin{tabular}{|c|l|l|}
\hline Setor & Experiência de uso de & Voluntariedade de uso de sistemas de informação: \\
- & sistemas de informação: & - Não gosto de usar os sistemas, prefiro papel \\
Idade & - Diariamente & - Não gosto de usar os sistemas, prefiro o Excel \\
- & - Frequentemente & - Às vezes prefiro usar os sistemas, às vezes prefiro Excel \\
Sexo & - Eventualmente & - Prefiro usar os sistemas, mas às vezes prefiro o Excel \\
- & - Raramente & - Prefiro usar sempre os sistemas \\
Feminino & - Nunca & \\
- & & \\
Masculino & &
\end{tabular}

Fonte: Adaptado do roteiro de entrevistas utilizado por Marques et al. (2018).

Vale registrar que a técnica de coleta de dados projetada inicialmente para esta pesquisa eram as entrevistas em profundidade (a exemplo do trabalho citado). Entretanto, verificou-se que a concorrida agenda das chefias de nível médio e alto impedia (ou dificultava 
muito) a abordagem síncrona e esses gestores sugeriram o uso do e-mail para poderem responder "quando tivessem um tempo". Apesar disso, 6 respondentes puderam ser entrevistados por telefone, o que permitiu aprofundamento dos significados.

As respostas $(E 1, \ldots, E 18)$ foram transcritas e tabuladas em uma planilha eletrônica (disponível sob consulta), permitindo tratamento em profundidade dos retornos. Observou-se que a maioria das respostas escritas (e faladas por telefone) apresentou rico detalhamento na análise da questão proposta e em implicações para a intenção de uso e o andamento do projeto de implantação. Grande parte teceu comparações com o sistema legado (dificuldades a serem superadas, por exemplo) para exprimir suas expectativas quanto ao sistema novo.

Foi definido, como unidade de análise, o projeto de implantação do sistema de controle financeiro. Vale registrar que, apesar do uso de um modelo de pesquisa quantitativa, uma análise quantitativa dos resultados seria equivocada e não teria validade estatística, dado o tamanho da amostra, mesmo considerando sua representatividade para o conjunto de key-users. Pensando nisso, uma análise qualitativa e interpretativa foi aplicada aos dados para a caracterização dos elementos componentes de cada construto no caso em estudo, fazendo uso da profundidade identificada nas respostas.

\section{Resultados}

O perfil dos respondentes mostra mulheres (9) e homens (9) (50\%-50\%), que estão nas seguintes faixas etárias: 18-20 anos (1), 21-30 (1), 31-40 (4), 41-50 (8) e 51-70 (3). Essa distribuição demográfica representa com expressiva semelhança a população total da empresa, que tem cerca de $50 \%$ de mulheres e $50 \%$ de homens e que cuja maioria dos funcionários está na faixa dos 40-50 anos, com uma minoria de menores de 30 anos.

Os respondentes atuam nos setores ou processos de controle financeiro (7), recuperação de créditos (4), tecnologia de informação (2), cobrança (1), tesouraria (1), contabilidade (1), auditoria e compliance (1) e recursos humanos (1), o que apresenta representatividade de todos os processos a serem atendidos pelo sistema.

Os respondentes usam os sistemas diariamente (15) ou frequentemente (3) e têm como preferência usar: sempre os sistemas (7), os sistemas, mas, às vezes, o Excel (8); ou às vezes os sistemas e às vezes o Excel (3). Esse perfil ilustra a experiência de uso dos sistemas pelos usuários-chaves contatados, junto de alguma preferência pelo Excel.

Para caracterizar cada variável do modelo, os resultados são mostrados organizados em um quadro para cada construto: 
Figura 3 - Expectativa dos respondentes de desempenho com adoção do sistema

\section{Expectativa de desempenho}

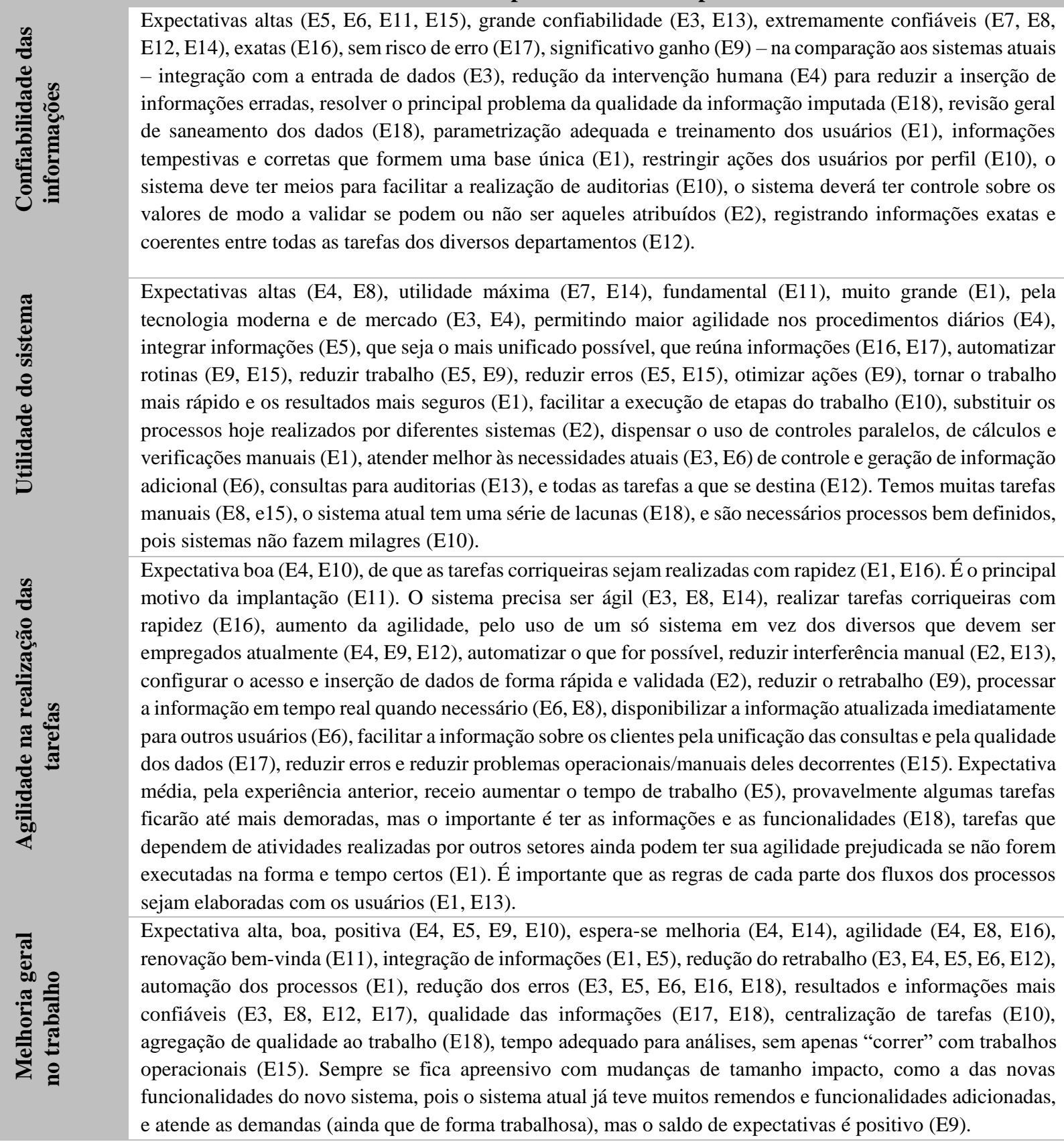

Fonte: Coleta e análise de dados.

Para a expectativa de desempenho (Figura 3), as respostas evidenciam expectativas altas para confiabilidade das informações, utilidade do sistema (comparado ao atual), agilidade na realização das tarefas (destacando definição de processos e colaboração entre áreas) e melhoria geral no trabalho (que reúne as melhorias citadas e causa apreensão). 
Figura 4 - Expectativa dos respondentes de esforço para adoção do sistema

\section{Expectativa de esforço}

\begin{tabular}{|c|c|c|}
\hline & 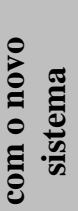 & $\begin{array}{l}\text { Esforço normal para adaptação a um sistema novo: dedicação (E3, E14), mudança gradativa e bem planejada } \\
\text { (E9), que a resistência à mudança não trave a implantação (E18), o esforço para interagir não deve ser um } \\
\text { empecilho (E1). Esforço grande (E11), deverá levar mais tempo que o previsto (E5). Redução considerável } \\
\text { no esforço (E4, E7, E8, E13), simples e funcional (E2), intuitivo, fácil (E6, E10), com interface amigável } \\
\text { (E15, E12, E16, E17, E18). }\end{array}$ \\
\hline 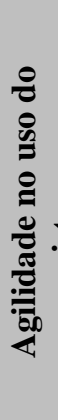 & & $\begin{array}{l}\text { Que o esforço seja baixo, por recebermos o tipo e tempo de treinamento necessário (E4, E14), menos esforço } \\
\text { que atualmente (E12). Que o sistema seja amigável (E18). Que tenha telas simples, organizadas, completas, } \\
\text { com destaque para informações importantes, para a interação ser ágil e eficaz (E2). Que tenha acessos } \\
\text { claramente definidos conforme atribuição de cada função (E6). Que seja intuitivo e com atalhos e } \\
\text { concentração de informações, sem necessidade de acessar muitos menus ou muitas trocas de páginas ou } \\
\text { diversas consultas paralelas para encontrar o que se procura (E8, E16, E17). Que automatize o máximo de } \\
\text { tarefas para que se tenha agilidade nas análises e reflexos positivos em outros setores (E15). Se o sistema } \\
\text { for claro e intuitivo, a agilidade será alcançada sem grandes problemas (E1). A agilidade virá de forma } \\
\text { natural, demandará tempo e paciência (E9), agilidade será boa depois de um tempo, até o pessoal se habituar } \\
\text { (E10), maior esforço concentrado no início até o domínio total das ferramentas (E11, E6). }\end{array}$ \\
\hline 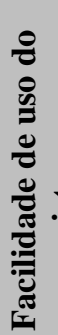 & & $\begin{array}{l}\text { Expectativas altas (E8), acredito que será muito fácil de usar (E7), expectativa boa, espero que não tenha } \\
\text { tantas telas (E10), teremos ganhos e avanços em relação ao sistema atual, por exemplo nos formatos de } \\
\text { relatórios (E9, E15), que o esforço seja baixo, por ter tido o treinamento necessário (E4). O sistema deve } \\
\text { apresentar facilidades (E14), ser amigável (E10, E12, E16, E17, E18), intuitivo (E1, E2, E6), "esperto", com } \\
\text { manuais para novos usuários (E1), com consulta e alteração de alçadas de acesso adequadas (E6). } \\
\text { Expectativa média, pela experiência anterior, receio que o novo sistema não seja amigável nem flexível (E5). } \\
\text { Expectativa de mais dificuldade no início, mas depois normaliza (E11). }\end{array}$ \\
\hline$\frac{\substack{0 \\
\frac{0}{2}}}{2}$ & $\begin{array}{l}\frac{2}{2} \\
\frac{0}{2} \\
\frac{2}{2} \\
\frac{2}{2}\end{array}$ & $\begin{array}{l}\text { Que seja baixo ou mínimo (E1, E2, E4, E7, E16, E17), por ser amigável (E15, E16, E18) e pela tecnologia } \\
\text { empregada (E4) e pelo treinamento proporcionado (E4, E6, E7, E9, E12, E14). O sistema atual é difícil, } \\
\text { acredito que melhore (E8). Grau de dificuldade normal (E11), médio (E3), ou razoável, pois processos } \\
\text { necessitarão ser revisados (E10), "faz parte" (E13), é só uma questão de adaptação do pessoal (sempre há } \\
\text { uma resistência às mudanças) (E15). Podem surgir grandes dificuldades em adaptar situações extraordinárias } \\
\text { (E1) e na agenda de treinamentos (E9), e não podemos regredir em controles e fluxo (E13). }\end{array}$ \\
\hline
\end{tabular}

Fonte: Coleta e análise de dados.

A expectativa de esforço (Figura 4) teve respostas variadas: alguns dizem esperar um esforço "normal" para a interação com o novo sistema; uns, um esforço "grande"; e outros, uma "redução considerável" no esforço (relativa ao esforço para interação com sistema atual). Quanto à agilidade no uso do sistema, a expectativa de alguns é "menos esforço que atualmente" e de uns é "maior esforço no início". Vários outros mencionam características que esperam que o sistema apresente para que haja essa agilidade. Em relação à facilidade de uso do sistema, há expectativas "altas" e "boas" por parte de alguns, "média" e "maior no início" para outros, "ganhos e avanços em relação ao sistema atual" para outros. Vários citam novamente características que esperam do sistema para que seu uso seja fácil. Também se 
dividem as respostas no que diz respeito à dificuldade em aprender: há expectativas de que o esforço seja "baixo", "mínimo"; "normal”, "médio", "razoável”; e "podem surgir dificuldades”.

Figura 5 - Influência social para adoção do sistema segundo os respondentes

\section{Influência social}

As melhores possíveis (E16), sensatas e motivadoras (ou desmotivadoras) importantes quanto à aceitação do
sistema (E1), apoio e incentivo no uso do sistema (E2), a opinião geral é de que o novo sistema vai melhorar
o desempenho das atividades (E12), consciência da importância dessa mudança para a empresa, para
modernização, solução de problemas e desempenho operacional (E15), adaptação ao novo sistema (E10),
patrocínio do sistema independentemente das dificuldades, que sempre vão existir (E18), tentar ver o lado
bom e mostrá-lo aos incrédulos (E11), haverá certamente opiniões de todas as formas, que podem contribuir
ou atrapalhar no andamento dos trabalhos (E5), sempre vai haver pessoas relutantes à quebra de paradigmas,
mas é importante ouvir e analisar as críticas construtivas (E13), pouco influenciam as opiniões em questões
práticas e objetivas como é o uso do sistema (E9, E14), não há espaço para pessoas defenderem o não uso de
um sistema no financeiro (E6).

Fonte: Coleta e análise de dados.

Em relação à influência social (Figura 5), as respostas parecem demonstrar certa aprovação geral e aceitação do novo sistema. A opinião das pessoas influentes ficou caracterizada como sendo positiva (o que reforça o resultado quanto ao construto expectativa de desempenho) e o fator "adaptação ao novo sistema" é mencionado, bem como atitudes a serem tomadas diante de "dificuldades", "incrédulos", "relutantes", "críticas" e "opiniões que podem atrapalhar". O quesito prestígio das pessoas que usam o sistema parece não ser bem entendido pelos respondentes: apenas uma terça parte responde que "não deverá ter influência" e "grande prestígio para todos que usam o sistema"; os demais, "não entendi". Os comentários quanto ao auxílio do gestor variam conforme o setor/departamento de atuação da pessoa (e, portanto, seu gestor), havendo, assim, de um lado, "expectativa muito positiva", de "apoio máximo, total e irrestrito"; de outro, "preocupação" e "novidade". No ponto apoio geral da empresa as considerações também se dividem: há "alta expectativa" e "apoio total", assim como 
“preocupação" e "resistência à mudança na cultura organizacional”.

Figura 6 - Condições facilitadoras da adoção do sistema segundo os respondentes

\section{Condições Facilitadoras}

Muito boas, os usuários têm sido consultados e envolvidos na fase de escolha do sistema, e mantidos atualizados através de e-mail, desde o início, das fases, dificuldades e benefícios de cada opção de sistema estudada, e assim, a escolha deverá atender também à participação dos usuários e devem se sentir mais confortáveis com as decisões tomadas a respeito da escolha (E1), altas, com a colaboração e o envolvimento das áreas e da T.I. para buscar o que já há de melhor (E9, E11, E15). E, dadas as condições de empresa pública: critérios incluídos no edital de concorrência (E4), processos bem definidos (E10), critério e foco na eficiência e usabilidade (E2), critérios de agilidade e na realidade atual de mercado (E16), critérios para que se escolha o sistema que atenda perfeitamente as necessidades (E12), suporte de consultoria especializada e contato com empresas parceiras e fornecedores (E3).

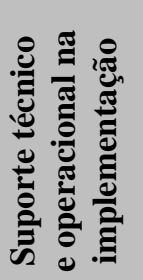

Expectativa alta (E2, E5, E11, E14), critério incluído no edital de concorrência (E4), solução rápida de problemas (E6, E12), total conhecimento do sistema e compreensão do negócio (E7, E8), profissionais qualificados (E18), suporte de qualidade, muito necessário especialmente no início (E10), suporte esclarecedor (E17), acompanhamento do fornecedor para efetiva implantação do sistema nas tarefas (E3, E12). E há a preocupação em aprender com os erros (E18), realizar a migração da base de dados com suas peculiaridades (E1), garantir o investimento em suporte amplo para autonomia (E9), e que seja realizado um trabalho sério com a máxima dedicação (E15).

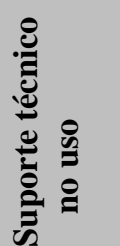

Alta expectativa (E5, E11), alta capacitação técnica (E2), rápido (E1,E7, E11, E16, E17, E18), amplo (E14), eficiente (E7), aspecto incluído no edital de concorrência (E4), atencioso (E15), à disposição para resolver todos os problemas (E14), totalmente adequado e aderente (E16), menu de ajuda e call center (E8), equipe especializada, que domine rotinas diárias e impactos de processos internos (E1, E6), suporte continuado do fornecedor e preparo necessário da equipe interna de T.I. (E12), acompanhamento próximo para detectar necessidades de pessoal técnico especializado (E3). E há preocupação quanto à repetição de experiências passadas em que o suporte se mostrou insuficiente (E9), importância da não acumulação dos problemas, ainda que a melhor solução demore (E1, E10), e necessidade de paciência e entendimento do processo de grande mudança (E15).

Fonte: A autora.

As respostas quanto às condições facilitadoras (Figura 6) manifestam expectativa “alta”, mas também “preocupação". Para o suporte para a escolha do sistema, a preparação do edital de concorrência conta com o envolvimento de usuários, de áreas de negócio e de T.I. e “critérios" relevantes e "processos bem definidos", além do apoio de consultoria e contato com parceiros e fornecedores, o que resulta em expectativa "alta". Quanto ao suporte técnico e operacional na implantação, também é "alta" a expectativa, sendo esperada a "solução rápida de problemas", embora haja mais de uma "preocupação": com a "migração", com o "investimento" e com o "trabalho sério com a máxima dedicação". Da mesma forma, a perspectiva quanto ao suporte técnico no uso do novo sistema suscita expectativa "alta", com um terço das respostas mencionando que espera que seja "rápido"; também tem-se a esperança de que haja uma equipe qualificada para a tarefa ("alta capacitação técnica", "equipe especializada, que domine rotinas", "necessidades de pessoal técnico especializado"); e há a preocupação com a "repetição de suporte insuficiente", com a "não acumulação de problemas" e com a "necessidade de paciência e entendimento do processo de grande mudança". 
Um resumo das respostas, em termos de uma avaliação "positiva", "negativa", "equilibrada" (que percebe pontos positivos e pontos negativos ao mesmo tempo) e "sem opinião”, é mostrada na Figura 7. Percebem-se, de maneira geral, opiniões positivas para todas as variáveis do modelo UTAUT (expectativa de desempenho, expectativa de esforço, influência social), o que pode influenciar na intenção de uso (que, portanto, parece positiva), e para as condições facilitadoras, o que pode intervir no comportamento de uso, a ser avaliado em pesquisas pós-implantação. Vale destacar a expressiva manifestação de preocupação quanto à expectativa de esforço como um ponto de atenção na condução do projeto, o que deve direcionar decisões com foco em treinamentos.

Figura 7 - Resultados da análise da pesquisa de opinião quanto às variáveis do modelo UTAUT nesta pesquisa

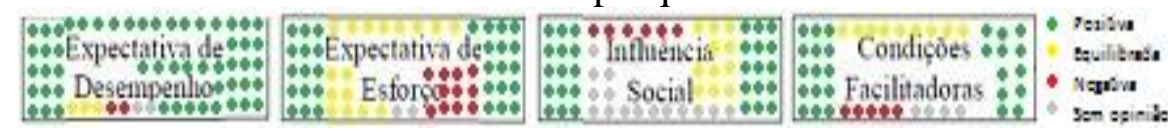

Fonte: A autora.

Sistematizando os resultados quanto às variáveis moderadoras, percebe-se na análise: os mais jovens (idade) parecem ter expectativas mais altas de confiabilidade (expectativa de desempenho); os mais velhos, mais baixas. Mulheres (gênero) parecem ter expectativa alta de aumento da agilidade; homens, não muito alta. Mais respondentes mais velhos (idade) parecem ter expectativas de baixo esforço necessário para ter agilidade no uso do sistema ("sistema intuitivo"). Os novatos (experiência) parecem ter expectativas de baixo esforço necessário para ter agilidade no uso do sistema ("sistema amigável”) e os mais experientes parecem ter expectativas entre "esforço baixo", "depende" e "difícil no início". A maioria dos mais experientes parecem crer que haverá facilidade de uso do sistema, poucos disseram "difícil no início" e "depende". Quanto aos menos experientes, um terço espera que seja fácil; outro terço, difícil. Dos mais jovens (idade), apenas um terço parece ter expectativa de auxílio do gestor da área, enquanto outro terço tem "preocupação"; dos mais velhos, metade espera esse auxílio (só dois têm baixa expectativa). A maioria dos mais jovens (e minoria dos mais velhos) (idade) parece ter expectativa de que haja prestígio das pessoas e alguns dos mais velhos opinam que isso não influencia. De todos os que usam sempre os sistemas, um terço dos que preferem usálos (outro terço crê que não influencia) e a maioria dos que usam às vezes (voluntariedade) parecem ter expectativa de que haja prestígio das pessoas. A maioria das mulheres (e minoria dos homens) (gênero) parece ter expectativa de apoio na opinião das pessoas que influenciam seu comportamento e a maioria dos homens (e minoria das mulheres) diz que essas opiniões podem contribuir ou atrapalhar. A maioria dos mais jovens (idade) pontua que essas opiniões 
importam (minoria diz que não); em relação aos mais velhos, metade espera opiniões de apoio e a outra metade comenta que essas opiniões podem contribuir ou atrapalhar. Os novatos (experiência) dizem que as opiniões podem contribuir ou atrapalhar; por sua vez, metade dos veteranos espera opiniões de apoio e metade diz que elas podem contribuir ou atrapalhar. Identificou-se diferença apenas da variável moderadora idade em relação à variável independente influência social (três quesitos com diferença e um sem). As condições facilitadoras, que influenciam não a intenção de uso, aqui focada, mas o comportamento de uso, são tema de pesquisas futuras pós-implantação do sistema. Percebem-se, nos resultados qualitativos desta pesquisa, as variáveis independentes que, segundo UTAUT, contribuem para a intenção de uso e uma de suas moderadoras, o que parece adequar-se apenas parcialmente às previsões do modelo.

Como mencionado, a organização pesquisada está em fase de seleção e implantação de um sistema e as regras para empresas públicas limitam as possibilidades de conhecimento do sistema pelos respondentes a, por exemplo, demonstrações de ferramentas de diversos fornecedores para os processos que compõem o escopo do edital de seleção do sistema. As expectativas dos respondentes parecem ser majoritariamente otimistas, embora pontuadas por ressalvas quanto à possibilidade de resistência à mudança e de insuficiência do suporte técnico. As projeções de desempenho e de esforço de vários respondentes são comparativas aos sistemas atuais e a uma recente tentativa de sistema novo (“creio que não possa piorar").

Nesse sentido, as expectativas de desempenho mostram a vontade/necessidade de ter inúmeros atributos no sistema a ser implantado, além daqueles do sistema atual e diferentes daqueles do sistema anteriormente tentado. Esse resultado parece ter relação com resoluções de pesquisas sobre sistemas financeiros, as quais identificaram que o design e a segurança são importantes para o desempenho das funcionalidades do sistema (Lai, 2017). As expectativas de esforço mencionam a melhoria em relação ao sistema atual e também a previsível maior dificuldade no começo. Os quesitos da influência social (quando entendidos) direcionam o julgamento do respondente para colegas/chefias e neles são percebidos focos de potencial dificuldade ("resistentes à mudança são os outros") em meio à opinião geral positiva quanto à migração para o novo sistema. Esses dados se parecem com os de pesquisas sobre sistemas financeiros e contábeis, as quais pontuam que se pode enfrentar resistências internas por falta de confiança ou conhecimento, aceitação de rotinas e insegurança (Angonese, \& Lavarda, 2014).

Os respondentes percebem potencial de alto desempenho do sistema e baixo esforço para usá-lo. Eles têm opinião geral positiva (ou seja, construtos que influenciam a intenção de 
uso) e parecem indicar impacto positivo. Ou seja, de acordo com outras pesquisas sobre sistemas financeiros, a facilidade de uso e a utilidade percebida contribuem para a efetiva intenção de uso do novo sistema (Lai, 2017). Algumas pessoas, entretanto, lembram de condicionar a expectativa de esforço às qualidades inerentes ao sistema, tais como usabilidade. Esse dado parece ter relação com a comparação com o sistema tentado, cujo fracasso pode ser atribuído a fatores como dificuldade de uso (expectativa de esforço alto), deficiências de funcionalidades (expectativa de desempenho baixo) e, consequentemente, não aprovação pelos formadores de opinião (influência social negativa).

Já as condições facilitadoras parecem gerar expectativas que, se cumpridas, têm influência no futuro comportamento de uso dos respondentes. Dentro dos limites impostos pelas condições específicas das empresas públicas, o suporte para escolha do sistema parece estar sendo aprovado. Por outro lado, manifesta-se grande expectativa pela qualidade e rapidez do suporte técnico e operacional da implantação e do suporte técnico do uso, que não se constatam na implantação do sistema tentado e geram queixas na situação atual.

De maneira geral, os mesmos fatores relatados em outros casos de pesquisas recentes sobre o tema são mencionados como preocupação para a efetiva adoção do sistema novo. A resistência à mudança cultural da organização, a desmotivação e o desinteresse com o projeto, a dificuldade para aprender a manipular a ferramenta e o treinamento e o suporte técnico deficientes são os pontos de atenção que ressoam com os exemplos de insucesso. Nesse sentido, podem ser citados os casos de adoção de tecnologia por produtores de café (Santos, \& Jesus, 2005) e de difusão de plataforma para comunidades virtuais de negócios (Hoppen et al., 2017), além das dificuldades em startups de Internet of Things (Bozzo et al., 2019) e dos sistemas financeiros e contábeis especificamente (Lai, 2017; Angonese, \& Lavarda, 2014).

Destaca-se, entre os resultados, uma ausência importante. Não é mencionado o fator “expectativas não realistas" em relação ao novo sistema, comum nos estudos relatados. Essa inexistência talvez surpreenda, já que esta é uma pesquisa sobre expectativas, específica da fase pré-adoção do novo sistema. Pode-se refletir sobre o próprio tema da pesquisa, resgatando um fator que potencialmente só venha a se efetivar e ficar evidente depois que os respondentes começarem a usar e conhecer melhor a ferramenta, quando puderem, então, perceber e ajustar suas expectativas.

\section{Conclusão}

Esta pesquisa atingiu seu objetivo de identificar, no contexto de uma empresa pública 
do setor financeiro, quais são as expectativas dos usuários-chaves em relação à implantação de um novo sistema integrado de informações para controle financeiro, analisando-as segundo o modelo UTAUT. Este último foi utilizado como direcionador do protocolo de coleta de dados e como fundamento de análise dos resultados, permitindo caracterizar as percepções dos respondentes quanto aos construtos expectativa de desempenho, expectativa de esforço, influência social e condições facilitadoras.

Pode-se concluir que as percepções dos respondentes em relação aos construtos são otimistas, podendo ser caracterizadas como: expectativa de alto desempenho, expectativa de mínimo esforço, influência social de apoio geral e condições facilitadoras de alta qualidade. Mas também foram mencionadas pelos respondentes: a resistência (de outras pessoas) à mudança na cultura organizacional, assim como sua preocupação com a qualidade do suporte técnico a ser prestado e a perspectiva de esforços maiores para aprendizagem no início da implantação.

O foco da análise deste trabalho está nas expectativas dos respondentes e em sua intenção de uso, não ainda no efetivo comportamento de uso, pois o sistema em questão encontra-se em fase de seleção para futura implantação. Nas etapas posteriores, a participação dos usuários e a gestão da mudança, inserida no escopo da gerência do projeto, serão focos de atenção relativos à aceitação da tecnologia. A avaliação do comportamento de uso configura, portanto, uma possibilidade concreta de pesquisa futura.

Vale ressaltar que, entre os fatores de sucesso de projetos de implantação de sistemas, constam o envolvimento dos key-users, o planejamento adequado e as expectativas realistas quando se fala em sistemas financeiros integrados, sabe-se que é baixa probabilidade de eles atenderem todos os requisitos e expectativas dos usuários, ocorrendo discrepâncias entre os anseios das pessoas e as funcionalidades do sistema. Assim, percebe-se que o processo de mudança para um novo sistema pode sofrer resistência de usuários com baixa intenção de uso, por rejeição, dúvida, medo, ansiedade, falta de confiança, inércia, etc. Por isso, essas expectativas devem ser monitoradas/gerenciadas no processo de mudança planejado/organizado, que explicite as funcionalidades reais do sistema, eliminando incertezas (e, assim, minimizando os receios e as rejeições por parte dos usuários-chaves envolvidos) e tratando das discrepâncias com cuidado, seja mudando o sistema ou mudando os procedimentos da empresa (ou adotando controles paralelos) (Souza, \& Saccol, 2011).

Nesse sentido, pesquisas sobre expectativas, intenção de uso e aceitação da implantação de um novo sistema de informação estão entre os fatores que podem contribuir para o sucesso do projeto. A realização desse tipo de estudo entre os funcionários, especialmente com usuários- 
chaves da ferramenta a ser implantada, pode configurar uma contribuição de interesse para a prática da comunidade profissional na forma de uma proposta de recomendação para empresas vivenciando situação semelhante, que podem replicar essa avaliação de expectativas, especialmente para sistemas financeiros e contábeis.

Os resultados desta pesquisa foram apresentados para os key-users e para os demais futuros usuários do novo sistema na empresa estudada. Os dados levantados estão sendo utilizados para direcionar decisões de projeto, tais como o sequenciamento da implantação, as necessidades de treinamento, a estratégia do processo de comunicação e o tom das reuniões com stakeholders, contribuindo para o eficiente gerenciamento de expectativas (tratamento próativo, alinhamento, ajuste, direcionamento) e reforço da intenção de uso.

Evita-se, com isso, o desperdício de recursos investidos na implantação de sistemas que não atendem às expectativas das pessoas e que, portanto, não contam com a sua intenção de uso, acabando subutilizados, o que representa custo para a empresa. Está previsto que esta pesquisa seja repetida em outras fases da implantação do sistema na empresa para a validação das percepções quanto aos módulos então já implantados e para a avaliação das expectativas quanto às funcionalidades que ainda serão instituídas nas demais etapas. Também se configura uma contribuição, na aplicação de um modelo já validado de avaliação para adoção de tecnologia, para um dos temas mais pesquisados na área de inovação e projetos, que é a aplicação de tecnologias, a qual apresenta desafios como este para uma gestão inovadora.

\section{Referências}

Angonese, R., \& Lavarda, C. E. F. (2014). Análise dos fatores de resistência envolvidos no processo de mudança no sistema de contabilidade gerencial. Revista de Contabilidade $e$ Finanças, 25(66), 214-227.

Bozzo, A. L., Freitas, H. M., \& Martens, C. D. P. (2019). Main initial difficulties faced by IoT startups. Revista da Micro e Pequena Empresa, 13(2), 40-59. http://dx.doi.org/10.21714/1982-25372019v13n2p4059.

Gil, A. C. (2008). Métodos e técnicas de pesquisa social (6a ed.). São Paulo: Atlas.

Hoppen, N., Klein, A. C. Z., \& Rigoni, E. H. (2017). Sociomaterial practices: challenges in developing a virtual business community platform in agriculture. Brazilian Administrative Review, 14(2), el70006. https://doi.org/10.1590/1807-7692bar2017170006

Lai, P. C. (2017). The literature review of technology adoption models and theories for the novelty technology. Journal of Information Systems and Technology Management, 14(1), 2138. https://doi.org/10.4301/s1807-17752017000100002. 
Lunardi, G. L., Dolci, D. B., \& Dolci, P. C. (2017). Adoção de tecnologia da informação e sua relação com a gestão de negócios em micro e pequenas empresas (MPEs). Revista de Administração da UFSM, 10(5), 929-948.

Machado, F. J., \& Martens, C. D. P. (2015, maio). Sucesso na gestão de projetos: uma análise bibliométrica. Proceedings of the International Conference on Information Systems \& Technology Management, 12, 3154-3173.

Malanovicz, A. V. (2017, maio). Lessons learned in failure cases in ERP implementation in Brazil: a literature review. Proceedings of the International Conference on Information Systems \& Technology Management, São Paulo, SP, Brasil, 14.

Markard, J., Wirth, S., \& Truffer, B. (2016). Institutional dynamics and technology legitimacy: a framework and a case study on biogas technology. Research Policy, 45(1), 330-344. https://doi.org/10.1016/j.respol.2015.10.009

Marques, K. Z., Behr, A., \& Malanovicz, A. V. (2018, maio). Aceitação de um ERP no departamento contábil de uma empresa pública. Proceedings of the International Conference on Information Systems \& Technology Management, São Paulo, SP, Brasil, 15.

Nguyen, T. H., Newby, M., \& Macaulay, M. J. (2015). Information technology adoption in small business: confirmation of a proposed framework. Journal of Small Business Management, 53(1), 207-227.

Project Management Institute. (2017). Um guia do conhecimento em gerenciamento de projetos (Guia PMBOK) (6a ed.). Newton Square, PA: Autor.

Ren, M. (2019). Why technology adoption succeeds or fails: an exploration from the perspective of intra-organizational legitimacy. The Journal of Chinese Sociology, 6(21), 126. https://doi.org/10.1186/s40711-019-0109-x

Santos, M. E., \& Jesus, J. C. S. (2005). Fatores que comprometem a adoção de tecnologia de informação em empresas cafeeiras. Production, 15(3), 456-468.

Souza, A. C., \& Saccol, A. Z. (Orgs.). (2011). Sistemas ERP no Brasil: teoria e casos. São Paulo: Atlas.

Venkatesh, V., Morris, M. G., Davis, G. B., \& Davis, F. D. (2003). User acceptance of information technology: toward a unified view. MIS Quarterly, 27(3), 425-478.

Vilela, B. A., Alves, C. S. A., Macedo, R. C., Werneck, M., \& Ferreira, F. C. (2018). Um mapeamento empírico do campo de gestão de operações e logística. Revista de Administração de Roraima-UFRR, 8(1), 86-103.

Webster, A., \& Gardner, J. (2019). Aligning technology and institutional readiness: the adoption of innovation. Technology Analysis \& Strategic Management, 31(10), 1229-1241. https://doi.org/10.1080/09537325.2019.1601694. 